\title{
Automated Optic Nerve Analysis for Diagnostic Support in Glaucoma
}

\author{
${ }^{1}$ Jin Yu, ${ }^{1}$ Syed Sibte Raza Abidi, ${ }^{2}$ Paul Habib Artes, ${ }^{1}$ Andrew McIntyre, ${ }^{1}$ Malcolm \\ Heywood \\ ${ }^{1}$ Health Informatics Laboratory, Faculty of Computer Science, Dalhousie University \\ ${ }^{2}$ Dept. of Ophthalmology and Visual Sciences, Dalhousie University \\ sraza@cs.dal.ca
}

\begin{abstract}
The availability of modern imaging techniques such as Confocal Scanning Laser Tomography (CSLT) for capturing high-quality optic nerve images offer the potential for developing automatic and objective methods for supporting clinical decision-making in glaucoma. We present a hybrid approach that features the analysis of CSLT images using moment methods to derive abstract image defining features, and the use of these features to train classifiers for automatically distinguishing CSLT images of healthy and diseased optic nerves. As a first step, in this paper, we present investigations in feature subset selection methods for reducing the relatively large input space produced by the moment methods. Our results demonstrate that our methods discriminate between healthy and glaucomatous optic nerves based on shape information automatically derived from CSLT tomography images.
\end{abstract}

\section{Introduction}

Confocal Scanning Laser Tomography (CSLT), a modern eye imaging technique, captures 3-dimensional optic nerve images that can be analyzed, in an automatic manner, to provide support in the clinical care of glaucoma patients [1]. Yet, to date, most diagnostic tools require human intervention-a trained professional has to manually define the margins of the optic nerve (a process that is somewhat subjective in nature and highly dependent on training and expertise). Whilst CSLT image analysis has tremendous potential to improve the clinical care of glaucoma patients, current methods for image analysis fail to detect optic nerve damage sufficient accuracy [2].

In this on-going project, we are working towards the development of a data-driven glaucoma diagnostic support system (shown in figure 1) that features the automatic interpretation of CSLT images by (a) applying image processing techniques to derive image-defining data that can be applied to a suite of data mining algorithms; (b) selecting a subset of image features that exhibit optimal classification capabilities for distinguishing between healthy and diseased optic nerves, and between different subtypes of optic nerve damage; (c) inducing classification rules in order to provide domain experts a symbolic explication of the data and the inherent class structures.

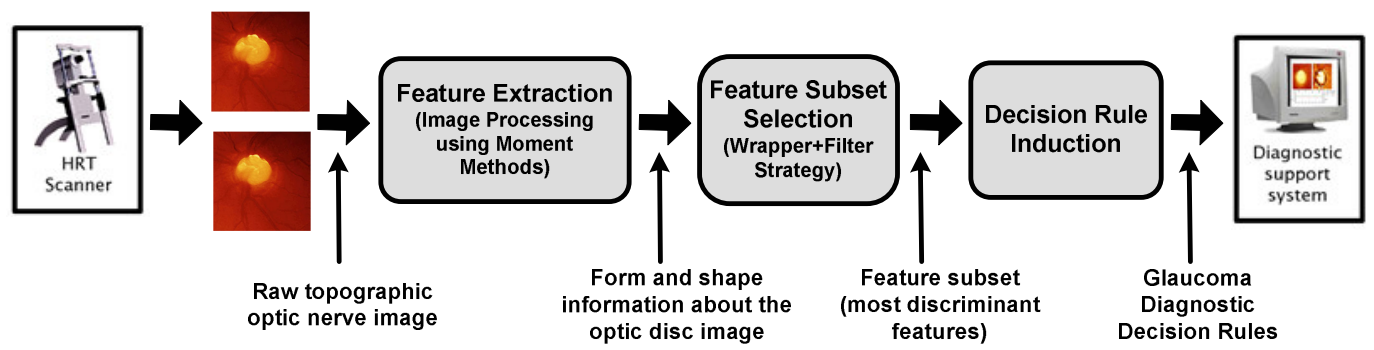

Figure 1. Functional design of a glaucoma diagnostic support system 
In this paper we present an automated approach to CSLT image analysis via feature subset selection leading to classification of the optic nerve images. First we present, the derivation of image-defining features from CSLT images using Moment Methods [3, 4]. Classification of CSLT images based on image features (or moments) is constrained by the relatively large input space-i.e. image features-produced by moment methods, thus prompting the need to applying feature selection methods $[5,6,7,8]$ to select a feature subset that offers optimal classification accuracy for classifying CSLT images of normal and glaucoma patient. We have developed a two-pass feature subset selection method that is a hybrid of wrapper and filter methods. In the first pass, wrapper models of Multilayer Perceptron (MLP) [9] and Support Vector machines (SVM) are used in a forward data selection manner to identify an optimal subset of lower order imagedefining moments that offer optimal classifications. In the second pass, the Markov blanket filter method [10] is used to select the highly relevant moments/features from the feature subset selected in pass 1. At the completion of the two feature selection passes we identify the smallest possible set of moments/features that provide the highest classification accuracy. Our results will demonstrate the efficacy of our automated approach to discriminate between healthy and glaucomatous optic nerves, based on shape information derived from CSLT topography images.

Analysis of optic nerve data, and CSLT based images in particular, using different feature subset selection and data classification methods has been actively pursued by researchers, with varying results [11-15]. Bowd et al [11], working with retinal tomograph images applied forward and backward feature selection methods for training MLP, SVM and linear discriminant functions; Park et al [12] have used correlation analysis and forward wrapper model to select features from optic nerve data for training SVM classifiers; Swindale et al [13] used a hill climbing wrapper model for feature selection to train SVM classifiers; whereas Cheng et al [14] and Peters et al [15] did not apply feature selection prior to their respective image analysis methods.

\section{Optic nerve image processing using moment methods}

The Heidelberg Retina Tomograph (HRT) is a CSLT system that uses a low-intensity monochromatic laser beam to scan the back of the eye sequentially in two dimensions to acquire a series of images from consecutive focal planes. Within each image series, the relative height of the retinal surface structure can be inferred by finding the focal plane in which maximum reflectance of each pixel occurs (topography image). After several image series for an eye are acquired (as shown in figure 2), the final mean topography images are used for diagnosis [1].

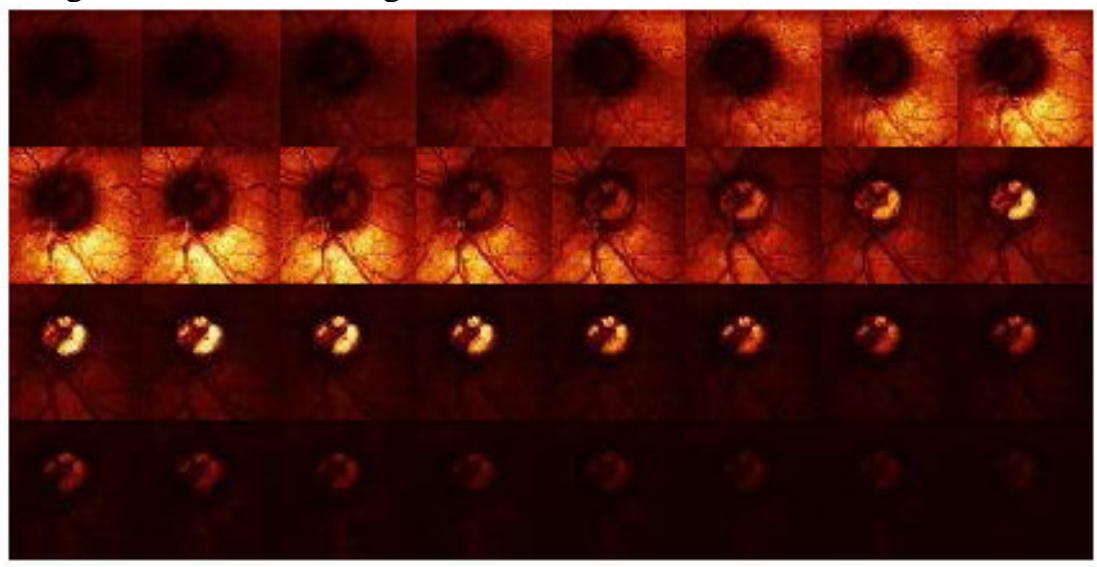

Figure 2. Confocal laser scanning image series of the optic disc 
We use an image processing technique, referred to as Moment Methods [3], to extract features from CSLT images. Moment features are properties of connected regions in binary images that are invariant to translation, rotation and scale and can describe the image content with respect to its axes and capture both global and detailed geometric information about the image.

In our work, we analyze CSLT images using Zernike moments [4] which use a set of complex polynomials which forms a complete orthogonal basis set on the unit disc $\left(x^{2}+\right.$ $\left.y^{2}\right)<=1$ (where $\mathrm{x}$ and $\mathrm{y}$ define the origin of the pixel). Put simply, Zernike moments describe the image's properties by their order $(n)$ and repetition $(m)$ with respect to a digital image - the low order moments capture gross shape information and high order moments incrementally resolve high frequency information (representing detail) of the digital image. Two attractive features of this analysis is that (a) moments can be made invariant to shifts, rotations and magnification changes; and (b) the optic nerve is centered in the image, thus avoiding the requirement for an independent segmentation stage in which the object is explicitly identified.

It should be noted that typically the low order moments capture fundamental geometric properties and high order moments represent detailed information of the image [4]. However, for image classification based on gross shape it can be argued that the high order moments do not contribute much information; in fact they can be regarded as noise. Given the above assumption, to classify CSLT images between normal and glaucoma, the task is to select an optimal number of lower order moments, however the problem is two-fold: (a) there is no available objective measure to determine the exact number of (low order) moments necessary for achieving high classification accuracy; and (b) there is no discernable relationship between the moments that can be utilized. Hence, there is a need for a feature selection strategy to objectively select an optimal set of moments, starting from the lowest order moments and moving towards higher order moments.

\section{Hybrid feature subset selection strategy for image-defining moments}

For our experiments we originally had 1257 tomography images taken at different time intervals from 136 subjects (51 healthy subjects and 85 glaucoma patients). For each CSLT image we generated 254 Zernike moments with order 1 to 29 . For the Zernike moments generated, the order $n$ and repetition $m$ meet the conditions $n-|m|=$ even and $|m|<=|n|$.

Given the set of 254 moments for each CSLT image, the objective is to determine a set of optimal moments that can provide high classification accuracy. The rationale for feature subset selection is based on the observation that a large number of abstract moments tend to compromise the accuracy of supervised learning classifiers, the classification rules are difficult to understand and the computational cost is high.

We have developed a hybrid feature subset selection strategy that combines both wrapper and filter models of feature subset selection, and operates in two phases. In the first phase, MLP and SVM based wrapper models are used to find an Optimal Moment Feature Subset (OMFS) which is the set consisting of low order moment feature groups that provide optimal data classification accuracy. In the second phase, a filter model based on a Markov Blanket (of the class label) [10] is applied to an inferred Bayesian network using the OMFS. The moments that have no causal relationship with the class are removed from OMFS to realize an even smaller feature sub-set of moments. In this paper, we present phase 1 of our feature selection strategy.

In the absence of any guiding principle to determine the size of the OMFS, we devised an accumulative feature selection strategy, whereby we incrementally add 
moments to an existing feature set and train a classifier (MLP and SVM) to determine the classification accuracy for the new feature subset. We had two options to generate the feature subset for training: (i) to add the next $N$ higher features to the existing dataset, where $N$ was deemed to range between 1-10 moments. Say, if $N=5$, then feature subset 1 would include moments $1-5$, feature subset 2 would add the next 5 moments to contain moments 1-10 and so on; or (ii) to use the intrinsic partitioning of the moments based on their order; the 254 moments were divided into 29 groups based on their order ranging from 1 to 29 . This implies that feature subset 1 includes moments with order 2 , feature subset 2 includes moments with order $2+3$, and so on. Both from a theoretical and experimental point of view, we determined that generating the accumulative feature subset based on adding moments of increasing order is the sound option.

Finally, to determine the size of the OMFS we generate 29 different feature subsets (in an accumulative manner), and for each feature subset we train two classifiers-MLP and SVM-and determine their classification accuracy. The classification accuracy trend for each of the 29 classifiers is plotted; the point on the plot (i.e. the moment group) from which the classification accuracy takes a downward trend (with the inclusion of the next higher moment group) is determined as the size of OMFS.

\section{Experimental results}

Two classifiers-i.e. MLP and SVM-were used as wrappers according to the abovementioned methodology to minimize the original set of moment features (totaling 254 moments) to a reduced feature subset. Determining the OMFS involved training the two classifiers with 29 different training sets created in an accumulative manner by adding the next higher moment group to the existing moment feature subset. All the moments were normalized into $[-1,+1]$ range.

For training the MLP we partitioned the feature subset-i.e. the data-into a training and test set. Different data partitions, ranging from $80 \%-20 \%, 75 \%-25 \%$ and $70 \%-30 \%$ (training\%-testing\%), were used. The classification accuracy results for MLP for all the moment groups are given in table 1. Figures 3 plots the classification trend for MLP.

For training the SVM, each candidate feature subset-i.e. the data-was divided into $75 \%$ training and $25 \%$ testing set. Based on the training data, a 5-fold cross validation was performed to find the optimal hyper parameters: $C$ and $\lambda$. Finally, the testing data was used to calculate the SVM's classification accuracy. In order to minimize the stochastic nature of the method, each candidate feature subset was trained 20 times and the mean classification accuracy is regarded as the final accuracy (table 1 and figure 3 ).

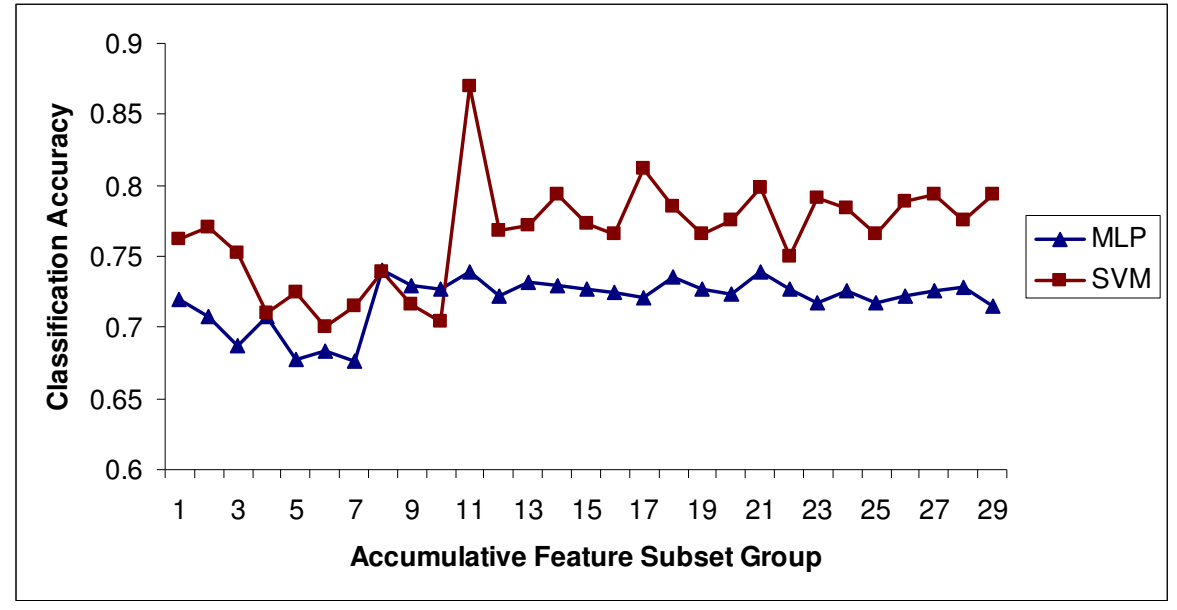

Figure 3. Classification accuracy for both MLP and SVM 
Table 1. Classification accuracy and standard deviation for MLP and SVM.

\begin{tabular}{|c|c|c|c|c|}
\hline \multirow{2}{*}{$\begin{array}{c}\text { Moment } \\
\text { Group }\end{array}$} & \multicolumn{2}{|c|}{ Multilayer Perceptron } & \multicolumn{2}{|c|}{ Support Vector Machine } \\
\hline & Accuracy & $\sigma$ & Accuracy & $\sigma$ \\
\hline 1 & 0.7197 & 0.0737 & 0.7618 & 0.0572 \\
\hline 2 & 0.7071 & 0.0735 & 0.7706 & 0.0724 \\
\hline 3 & 0.6868 & 0.0760 & 0.7529 & 0.0740 \\
\hline 4 & 0.7072 & 0.0661 & 0.7103 & 0.0823 \\
\hline 5 & 0.6769 & 0.0702 & 0.7250 & 0.0818 \\
\hline 6 & 0.6832 & 0.0730 & 0.7000 & 0.0736 \\
\hline 7 & 0.6762 & 0.0852 & 0.7147 & 0.0733 \\
\hline 8 & 0.7400 & 0.0685 & 0.7397 & 0.0590 \\
\hline 9 & 0.7297 & 0.0680 & 0.7162 & 0.0679 \\
\hline 10 & 0.7271 & 0.0609 & 0.7044 & 0.0914 \\
\hline 11 & 0.7393 & 0.0752 & 0.8696 & 0.0305 \\
\hline 12 & 0.7224 & 0.0668 & 0.7676 & 0.0464 \\
\hline 13 & 0.7324 & 0.0690 & 0.7721 & 0.0482 \\
\hline 14 & 0.7294 & 0.0647 & 0.7941 & 0.0446 \\
\hline 15 & 0.7268 & 0.0700 & 0.7735 & 0.0427 \\
\hline 16 & 0.7241 & 0.0829 & 0.7662 & 0.0661 \\
\hline 17 & 0.7210 & 0.0708 & 0.8117 & 0.0746 \\
\hline 18 & 0.7359 & 0.0713 & 0.7853 & 0.0652 \\
\hline 19 & 0.7272 & 0.0723 & 0.7662 & 0.0735 \\
\hline 20 & 0.7235 & 0.0925 & 0.7750 & 0.0751 \\
\hline 21 & 0.7390 & 0.0756 & 07985 & 0.0632 \\
\hline 22 & 0.7271 & 0.0961 & 0.7500 & 0.0667 \\
\hline 23 & 0.7170 & 0.0789 & 0.7912 & 0.0541 \\
\hline 24 & 0.7257 & 0.0726 & 0.7838 & 0.0632 \\
\hline 25 & 0.7176 & 0.0713 & 0.7662 & 0.0792 \\
\hline 26 & 0.7224 & 0.0874 & 0.7882 & 0.0562 \\
\hline 27 & 0.7257 & 0.0718 & 0.7941 & 0.0581 \\
\hline 28 & 0.7288 & 0.0723 & 0.7750 & 0.0659 \\
\hline 29 & 0.7144 & 0.0740 & 0.7941 & 0.0631 \\
\hline
\end{tabular}

A comparison of the classification accuracy trends for both the MLP and the SVM classifiers (see table 1 and figure 3 ) shows that both these classifiers have a similar classification accuracy trend-i.e. they both start with a relatively high accuracy with the first moment group and then the accuracy drops with the accumulation of the next few moment groups. But then the accuracy starts to pick up and for the MLP it peaks when the feature subset constitutes the first 8 moment groups, whereas for the SVM the accuracy peaks for the first 11 moment groups. The classification accuracy peak is not sustained for both classifiers with the inclusion of next higher moment groups; in fact the classification accuracy noticeably drops off with the inclusion of higher moment groups in the accumulative feature subsets. Furthermore, it can be observed that the classification accuracy with higher order moment groups is relatively low as compared to the peak achieved with the lower order moments.

Based on the above interpretation of the classification accuracy trend for both classifiers, we determined the OMFS to constitute the first 11 moment groups-i.e. the first 47 moment features-because the SVM exhibited the highest accuracy and the MLP exhibited the second highest accuracy with the first 11 moment groups.

\section{Conclusions}

We have presented an alternate approach to analyze CSLT images for glaucoma detection. In the next step we plan to derive symbolic rules using rule induction algorithms to provide symbolic knowledge for diagnosing glaucoma leading to the automation of decision support for glaucoma based on CSLT images. 
Table 2. Classification accuracy before and after feature subset selection

\begin{tabular}{|l|l|l|}
\hline \multicolumn{1}{|c|}{ Feature Subset Size } & \multicolumn{1}{c|}{ Classifier } & Accuracy \\
\hline All 254 moment features & MLP & $71.44 \%$ \\
\hline All 254 moment features & SVM & $79.41 \%$ \\
\hline 47 moment features in OMFS & MLP & $74.00 \%$ \\
\hline 47 moment features in OMFS & SVM & $86.96 \%$ \\
\hline
\end{tabular}

From a practical standpoint, in this paper we have demonstrated again the potential of using Zernike moment methods as a viable image-processing approach for working with CSLT optic nerve images [16]. Furthermore, we presented a novel feature subset selection strategy that allows us to minimize the feature space without the loss of information. Table 2 indicates that through the first pass of our feature subset selection strategy we managed to reduce the feature set from 254 moments to a much smaller feature subset comprising just 47 salient moments, whilst achieving a slight increase in the classification accuracy. The second pass of our feature subset selection strategy, involves the use of a Markov Blanket as a filter model to the 47 features and we are able to further minimize the feature set to just 6 most salient moments whilst maintaining the classification accuracy (results to be presented in a separate paper).

Acknowledgements: This research is supported by a Nova Scotia Health Research Foundation (NSHRF), Canada research grant titled "Interpreting Optic Disk Tomography in Glaucoma: Importance of Shape Information"

\section{References}

[1] G. Zinser, R.W. Wijnaendts-van-Resand, A.W. Dreher. "Confocal Laser Tomographic Scanning of the Eye", Proc. SPIE 1161, 1980, pp. 337-344,

[2] B.A. Ford, P.H. Artes, T.A. McCormick, M.T. Nicolela, R.P. LeBlanc, B.C. Chauhan. "Comparison of Data Analysis Tools for Detection of Glaucoma with The Heidelberg Retina Tomograph", Ophthalmology 110 (6) 2003, pp 1145-1150.

[3] Yu. V. Vorobyev, "Method of Moments in Applied Mathematics", Gordon and Breach Science Publishers, New York, 1965.

[4] C.H. Teh, R.T.Chin, "On Image Analysis by the Methods of Moments," IEEE Trans. Pattern Analysis by Machine Intelligence 10(4), July 1998, pp. 96-513.

[5] R. Kohavi, G. John, "Wrappers for Feature Subset Selection”, Artificial Intelligence Journal , Vol. 97 (1-2).

[6] D. Koller, M. Sahami, "Toward Optimal Feature Selection", Proc. of the $13^{\text {th }}$ Intl. Conf. on Machine Learning, 1996, pp. 284-292.

[7] I.Guyon, A. Elisseeff, "An Introduction to Variable and Feature Selection", Journal of Machine Learning research, vol. 3, 2003, pp. 1157-1182.

[8] A.L. Blum, P.Langley, "Selection of Relevant Features and Examples in Machine Learning", Artificial Intelligence, 1997, pp. 245-271

[9] A.Khotanzad, J. Lu, "Classification of Invariant Image Representations Using a Neural Network", IEEE Trans Acoustics, Speech, and Signal Processing, Vol. 38, 1990, pp. 1028-1038.

[10] E.R. Hruschka Jr., E.R. Hruschka, N.F.F. Ebecken, "Feature Selection by Bayesian Network", Canadian AI Conference, LNAI 3060, Springer Verlag, 2004, pp. 370-279.

[11] C. Bowd, K. Cban, L.M. Zangwill, M.H. Goldbaum, T. Lee, T.J. Sejnowski, R.N. Weinreb, "Comparing Neural Networks and Linear Discriminant Functions for Glaucoma Detection using Confocal Scanning Laser Opthalmoscopy of the Optic Disc," Investigative Opthalmology \& Visual Science, Vol. 43 (11), 2002.

[12] J. Park, J. Reed, Q. Zhou, "Active Feature Selection in Optic Disc Nerve Data using Support Vector Machine", IEEE World Congress on Computational Intelligence, 2002.

[13] N.V. Swindale, G. Stjepanovic, A. Cbin, F. Mikelberg, "Automated Analysis of Normal and glaucomatous optic nerve head topography images", Investigative Opthalmology \& Visual Science, Vol. 41 (7), 2000.

[14] S. Cheng, Y. Huang, "A Novel Approach to Diagnose Diabetes Based on the Fractal Characteristics of Retinal Images," IEEE Trans. on Information Technology in Biomedicine, Vol.7 (3), 2003.

[15] A.Peters, B. Lausen, G. Michelson, O. Gefeller, "Diagnosis of Glaucoma by Indirect Classifiers", Methods of Information in Medicine, 2003.

[16] A. McIntyre, M. Heywood, P. Artes, S.S.R Abidi, "Toward Glaucoma Classification with Moment Methods", 1st Canadian Conference on Computer and Robot Vision (CRV04), 2004, London, Ontario. 